\title{
Harmless Over the Counter Medications- are they Truly Harmless for the Elderly?
}

\author{
Vivian C. Barrera ${ }^{1}$, W. T. Chang ${ }^{2}$, Kuah Poh Kah ${ }^{3}$, S. C. Lim ${ }^{4 *}$ \\ ${ }^{1}$ Resident Physician, Department of Geriatric Medicine, Changi General Hospital, Singapore. \\ ${ }^{2}$ Principal Clinical Pharmacist, Department of Pharmacy, Ng Teng Fong General Hospital. \\ ${ }^{3} 4^{\text {th }}$ Year Medical Student, National University of Singapore, Yong Loo Lin School of Medicine, Singapore. \\ ${ }^{4 *}$ Adjunct Associate Professor, Senior Consultant, Department of Geriatric Medicine, Changi General Hospital, \\ Singapore. \\ *lim.si.ching@singhealth.com.sg \\ *Corresponding Author: Si Ching Lim, Adjunct Associate Professor, Senior Consultant, Department of Geriatric \\ Medicine, Changi General Hospital, Singapore.
}

\begin{abstract}
Drugs available over the counter are often considered to be harmless, as they can be self-prescribed for common ailments or taken as supplements. The range of drugs available over the counter is getting larger and the elderly are the biggest consumers of these presumptive harmless medications. However, the elderly have altered pharmacokinetics and pharmacodynamics and age related decline in physiological reserves putting them at higher risk of developing complications associated with medication usage. The long list of medications which most elderly take for their comorbid medical conditions also put the elderly at risk of developing complications. The use of over the counter medication may worsen the underlying medical conditions. Caution must always be exercised when considering a new drug for the elderly.
\end{abstract}

Keywords: acute kidney Injury, elderly, NSAIDS, antihistamines, H1 antagonists, benzodiazepines, adverse drug reactions

\section{INTRODUCTION}

The elderly population is increasing worldwide, and the elderly patients are often on a long list of medications which include over the counter medications and prescribed medications for their multiple comorbidities. The over the counter medications (OTC) are often viewed as harmless and are often self-prescribed. Qato showed that the older adults are the most frequent consumers of the OTC, with an average older adult taking 4 medications from the OTC. (1) The range of drugs available over the counter is rapidly increasing, with over 300,000 FDA approved drugs, available to the consumers in the US market. (2) Drug related problems resulting in adverse reactions, poorly controlled chronic diseases, delirium, etc have caused increased hospital visits at the emergency department, ambulatory setting and hospital admissions, resulting in high healthcare costs. (3) The combination of OTC medications and prescribed medications has a potential to cause drug-drug interactions or interfere with the existing medical conditions resulting in adverse events, among the elderly with reduced physiological reserve.

Adverse drug reactions (ADR) refer to the harmful reactions unrelated to usage of drugs as FDA approved drugs, under normal usage and dosage, including side effects, allergic reactions, toxic reactions, drug dependence, mutation, distortion, carcinogenic effects, etc (4). The possibility of an adverse drug event should always be borne in mind when prescribing to an older adult individual, as adverse events to common drugs can create serious consequences in elderly.

This paper gives a few examples of seemingly harmless OTC which are frequently self-prescribed for common ailments, without realising the potential adverse reactions which resulted in hospitalisations, prolonged hospitalisation and morbidity. A short review of these potentially harmful OTC medications is also summarised. 
Harmless Over the Counter Medications- are they Truly Harmless for the Elderly?

\section{Patient 1}

Madam W is a 91-year-old lady with a background history of dementia, depression, diabetes, chronic kidney disease, hypertension, hyperlipidaemia, NSAID gastropathy, osteoarthritis of the knees and an old right distal radius fracture following a fall. She lives in the community with her family and a full time caregiver.

Despite the long list of co-morbidities, she was not on any prescription medications for her chronic medical conditions. Madam W was complaining of right knee pain for 10 days, for which she consulted a family physician and was given Voltaren (Diclofenac) for a week. She was then brought back to the family physician 7 days later for lower limb swelling and persistent knee pain which did not improve. She was given Scanduretic (Amiloride/ Hydrochlorothiazide) for her lower limb swelling, additional Voltaren (Diclofenac) for another week and a prescription for Periactin (Cyproheptadine) 1 tab daily which was intended for improving her appetite, as her family complained that her appetite has been poor since the last week.

Two days after the second consultation, she was brought to the emergency department with complaints of drowsiness, functional decline and poor feeding. Her physical examination showed that she was drowsy and dehydrated with no other significant physical findings. The diagnoses were:

- Delirium due to cyproheptadine, poorly controlled pain, AOCKD, poor oral intake.

- Acute on chronic kidney disease (AOCKD) due to NSAIDS and Scanduretic use.

- Constipation and retention of urine due to Cyproheptadine, delirium and functional decline.

- Osteoarthritis of the left knee with flare.

Her initial investigations showed worsening of her kidney function with creatinine of $359 \mathrm{umol} / \mathrm{L}$ (baseline 165umol/L a year ago and urea of 20.8mmol/ (baseline $9 \mathrm{mmol} / \mathrm{L}$ ) and hyperkalaemia (K: $6.5 \mathrm{mmol} / \mathrm{L}$ ). Due to her prolonged immobility, she was also noted to have constipation and retention of urine, requiring intermittent catheterization. Constipation was cleared with suppository and simple laxatives. She was put on an IV drip with improvement of her kidney function and was back to baseline on the $4^{\text {th }}$ hospital day. All the medications prescribed by the family physician were ceased and her sensorium and function also improved. She was eating quite well in the ward, following improvement of her sensorium and her knee pain was managed with simple analgesia, heat pack and physiotherapy.

At discharge, her cognition has returned to baseline, answering questions appropriately and she was walking with a walking frame, with minimal supervision. She was weaned off intermittent catheterisation and was passing urine normally.

\section{Patient 2}

Madam Y is an 85-year-old Chinese female with past medical history of hypertension, hyperlipidaemia, diabetes mellitus, asthma, and previous fractures of pubic rami after a fall in 2017.

There was no documented history of dementia nor cognitive impairment prior to this admission. She was ADL independent, community ambulant, managing her housework and cooking at home. She was taking the following medications prior to admission, Atenolol, Amlodipine, Simvastatin, Mecobalamin, Lisinopril and Paracetamol for pain.

She was admitted in hospital for viral respiratory tract infection, acute kidney injury likely due to poor appetite, and giddiness. On admission, she was assessed to be alert and oriented to time, place and person. She claimed her dizziness resolved after cessation of her 3 antihypertensives. Along with her chronic medications, she was prescribed Dextromethorphan $10 \mathrm{ml}$ TDS for her cough, potassium supplement for hypokalemia, metoclopramide on PRN basis, calcium carbonate and cholecalciferol.

After her $4^{\text {th }}$ dose of Dextromethorphan, the staff reported that she kept standing up and was walking around her bedside and later kept going to toilet. She was unable to sleep with interrupted toilet visits on her second night in the hospital. The nurses assed her gait to be unsteady and she was irritable, shouting at the nurses when they told her to ask for help should she need to get out of bed. She was also reported to scold a patient next to her bed for being noisy at night.

Madam Y had an episode of near fall in the ward on the second night as she frequently attempted to ambulate in the ward, her gait was noted to be unsteady and 
Harmless Over the Counter Medications- are they Truly Harmless for the Elderly?

she appeared confused. She was put on upper limb restraints, which worsened her aggression, and she ended up kicking and biting the staff. She was then put on lower limb restraints. While the nurses were tying her down, she struggled to sit up, leaned across the bed and attempted to kick the nurses. She twisted her restrained left arm which resulted in a fracture of her left humerus.

Following the fracture, she was assessed by geriatricians to have hyperactive delirium due to Dextromethorphan. Further cognitive history from her family showed that she has been declining in her function due to worsening short term memory and was diagnosed to have mild Alzheimer Disease. She stayed in the acute hospital for 22 days.

\section{Patient 3}

Madam $\mathrm{T}$ is an 86-year-old lady with a past medical history of hypertension, dyslipidemia, hyperthyroidism (s/p thyroidectomy 1961), brain aneurysm (s/p coiling in 1998), left eye glaucoma and osteoporosis. She was brought to the hospital for worsening insomnia and one week's duration fluctuating behavioral changes (occasionally incoherent, paranoid with emotional outbursts). She has a background of insomnia for years and was on a combination of Hydroxyzine and hypnotics from her family physician. Her family reported worsening insomnia over the last 2 months prior to admission and low mood. She had trouble with her stock as they were losing their values and she had a recent fall out with her confidante. She was selftitrating her sleep medication at home.

Timeline of her medication changes: She was selfmedicating on hydroxyzine and her family was uncertain how much hydroxyzine she was taking to induce sleep. She visited the emergency department after 2 weeks for insomnia where she was given Zolpidem, Bromazepam and Hydroxyzine. This combination cocktail failed too. She subsequently went to see a psychiatrist and was diagnosed with major depression, treatment with Mirtazapine and Lorazepam was given. A week later, she was noted to have outbursts of inappropriate behavior at home, for which she was aggressive and threatened her helper at home. Her family suspected she was still self-medicating with Hydroxyzine in addition to the new prescription. Her family took her to see another physician, where Mirtazapine was stopped and she was started with Zopiclone. She was brought to emergency department the following day for persistent insomnia and noted to be incoherent.

Madam $\mathrm{T}$ has a baseline anxious personality and was the sole caregiver of her husband who has dementia. Her pre-morbid function level is high as she was investing in shares and was still driving. Her physical examination showed an anxious and agitated thin elderly lady who was incoherent. She was intermittently screaming, answering questions incoherently, not eating and not sleeping. There were no significant physical signs of note. Her parameters were all stable and she was afebrile. Her blood investigations showed acute kidney injury with low serum sodium and potassium levels, mild metabolic acidosis and nothing else. Septic work up were all negative. Electrocardiogram and chest Xray were unremarkable. CT brain and MRI brain with contrast were all unremarkable, with no evidence of stroke or signs suggestive of leptomeningeal diseases. Working diagnosis:

- Delirium due to medications with anticholinergic properties and acute kidney injury.

- Acute kidney injury due to dehydration and poor oral intake.

She was seen by psychiatry and neurology multiple times with the consistent impression of delirium with visual hallucinations. Extensive workup for organic causes which included Cerebrospinal fluid (CSF) examination for infective and autoimmune encephalitis were done and were all negative. CSF cytology and CSF NMDA were negative. CCSF cell count, glucose content and protein content were all unremarkable. CSF smear for TB and fungal were also negative. To exlude non-convulsive seizures, an electroencephalogram (EEG) was requested which showed mild diffuse encephalopathy with no evidence of seizure. Her abbreviated mental test score prior to admission was 8/10.

During her stay in the ward, she refused all food and drink. After extensive discussion with family, a nasogastric tube (NGT) was inserted to allow for administration of nutrition, medication and fluids. The indications for NGT were to get nutrition and hydration into her since poor nutrition is a cause for delirium and the tube will be removed as soon as she can sustain her nutritional needs with oral feeding. Due to her 
Harmless Over the Counter Medications- are they Truly Harmless for the Elderly?

agitation, she required mittens and intermittent arm restraints to stop her from pulling the NGT. During her stay, she refused to get out of bed and she developed acute retention of urine with febrile episodes requiring antibiotics for urinary tract infection. She also had an episode of lower gastrointestinal bleeding, which was due to haemorrhoids and settled spontaneously.

At admission, all her hypnotics were discontinued. She required the use of haloperidol $0.5 \mathrm{mg}$, up to a maximum of $1.5 \mathrm{mg}$ per day as needed for agitation. Her sleep was fragmented during the nights, scoring a max of 4 hours per night, but had intermittent naps during the day. She was started with Mirtazapine for her depression after $3^{\text {rd }}$ week of hospital stay as she was frequently crying and depressed. She was gradually noted to show signs of improvement in her cognition after 6 weeks of hospital stay. She had also severely declined in her function compared to her pre morbid level.

\section{Discussion}

Drug related problems are common in older adults, one of which is adverse drug effects. Adverse drug effects are effects that are unwanted, uncomfortable, or dangerous. Common examples are over sedation, confusion, hallucinations, falls, and bleeding (5). Adverse drug effects can occur in any patient, especially among the older adults who have reduced physiological reserves. The pharmacokinetics and pharmacodynamics are also different among the elderly due to impaired organ function, body fat / lean muscle mass distribution and comorbidities. In addition, the long list of prescription drugs can potentially cause drug-drug interactions resulting in adverse events. The authors have reported 3 cases of adverse drug reactions from the commonly used drugs which are widely available from over the counter. In Singapore where the authors practise, the range of drugs available from the OTC are not as wide as the US. The commonly available drugs like antihistamines, cough syrups still require a doctor's prescription

One in ten hospital admissions in older patients are due to adverse drug reactions. Non steroidal antiinflammtory drugs (NSAIDs) are the commonest class of medication causing hospital admissions. (6) One in eight elderly patients hospitalised for an adverse drug reaction had a repeat admission for an adverse drug reaction within 12 months of discharge. The commonest organ dysfunction related to adverse events from drug use is renal disorders (44.4\%) and the most frequently implicated drug classes were diuretics (44.8\%) and NSAIDS. (7)

NSAIDs are one of the most commonly prescribed pain medications. It is a highly effective drug class for pain and inflammation. However, NSAIDs are known for multiple adverse effects, including gastrointestinal bleeding, cardiovascular side effects, and NSAID induced nephrotoxicity (8). NSAIDs associated nephrotoxicity are more prominent and more severe in older adults, since their renal functions are subnormal. NSAIDs alter renal functions through their effects on renal prostaglandins leading to reversible renal ischemia and reduced GFR from afferent arteriorlar constriction. Other complications, though infrequent, related to NSAIDs usage include hypertension, salt and water retention resulting in oedema and hyperkalaemia remain a concern in patients who are at risk and can develop acute kidney injury (AKI). (9)

Age-related decline in renal blood flow among chronic hypertensive patients, concurrently with volume depletion due to dehydration or diuretics puts them at an even higher risk of developing renal complications with NSAIDs use. $(8,9)$ This was clinically evident in Madam W who is elderly, with long history of hypertension and chronic kidney disease. Prior to her emergency department visit, Madam W received Thiazide diuretic for lower limb swelling. Her plasma creatinine, urea and potassium were noted to be elevated much more compared to her baseline values. The course of NSAIDs given to her (Diclofenac $50 \mathrm{mg}$ three times a day, $150 \mathrm{mg} /$ day) was sufficient to precipitate acute renal failure as as it inhibits the synthesis of renal prostaglandin needed to keep the afferent arterioles open in the face of reduced renal perfusion associated with dehydration. According to the Naranjo adverse drug reaction probability scale, diclofenac is the probable cause of this hemodynamically mediated acute renal failure.

Hemodynamically mediated AKI due to NSAIDs among volume depleted patients is reversible and is mostly related to the dose and duration of exposure. (10) For Madam W, patient plasma creatinine and urea started to improve on 4th day of drug discontinuation with intravenous hydration and improved oral intake. 
Harmless Over the Counter Medications- are they Truly Harmless for the Elderly?

Non-steroidal anti-inflammatory drugs (NSAIDs) have also been reported to induce delirium as some NSAIDs can cross the blood-brain barrier. Indomethacin has been well reported to cause CNS suppression and contribute to delirium, Diclofenac has not been reported to do so. In Madam W's case, Diclofenac may not have contributed to delirium.

Madam W also received Cyproheptidine, an $\mathrm{H}_{1}$ antagonist, which is usually given for allergic conditions. Off label uses include decreased appetite due to chronic disease, serotonin syndrome, spasticity associated spinal cord damage. (21) Cyproheptadine is identified in the Beers Criteria as a potentially inappropriate medication to be avoided in patients 65 years and older (independent of diagnosis or condition) due to its potent anticholinergic properties resulting in increased risk of confusion, dry mouth, constipation, and other anticholinergic effects or toxicity; its use should also be avoided due to reduced renal clearance with advanced age and tolerance associated with use as a hypnotic. In this case, Cyproheptadine may have contributed to the patient's delirium, constipation and urinary retention.

Dextromethorphan (DXM) is a common remedy for cough, the dextrorotatory isomer of 3-hydroxy$\mathrm{N}$-methylmorphinan, is the main ingredient in a number of widely available, over-the-counter antitussives (12). Dextromethorphan is a highly effective and widely used nonopioid antitussive drug for upper respiratory tract infections. As it has been in use for more than 30 years, a large body of clinical experience has been used to formulate a safety profile. In humans, dextromethorphan lacks the pharmacological characteristics of opiate alkaloids. In normal individuals, at therapeutic doses, dextromethorphan is devoid of analgesic, euphoriant and physical dependence-producing properties. In animals, dextromethorphan is non-addictive, even in high experimental doses (13).

Its efficacy is similar to that of codeine. This agent crosses the blood-brain-barrier and activates sigma opioid receptors on the cough centre in the central nervous system, thereby suppressing the cough reflex. Unlike the other opiates, it has no analgesic power. Its toxicity is low and adverse reactions are rare, the most commonest of which being those that affect the central nervous system or psychiatric disorders; rash of urticaria type and anaphylaxis are totally rare. This drug can cause dizziness, drowsiness, nervousness, restlessness, gastrointestinal distress, nausea, stomach pain, vomiting, insomnia, ataxia, slurred speech and nystagmus to mood changes, perceptual alterations, inattention, disorientation and aggressive behaviour. (14).

At low dosages, it binds to the opioid receptor, which accounts for suppression of the cough reflex (15). At higher dosages it is metabolized to dextrophan, an $\mathrm{N}$-methyl-D-aspartate (NMDA) antagonist (16) that can produce dissociative hallucinations $(17,15)$, similar to phencyclidine and ketamine. In addition to dissociative effects, tachycardia, hypertension, agitation, ataxia, and psychosis have also been reported at those higher dosages (19-21). Our patient was given only 4 doses of $10 \mathrm{ml}$ but has already exhibited agitation and aggression.

Dextromethorphan is largely metabolised by the CYP P450 2D6 isozyme, which is associated with polymorphisms affecting its activity. Potentially, medications with strong inhibiting effect on this isozyme could increase the risk for adverse drug reactions to dextromethorphan (25). However, no such drug was found to have been taken by Madam Y. She also did not have liver impairment that could have reduced the clearance of the drug. Therefore, it is possible that she could be a poor metabolizer for the isozyme. Patients with such polymorphism have been shown to have poor tolerance of dextromethorphan. Nevertheless, it must be emphasized that the incidence of poor 2D6 metabolizer is found to be rare $(<1 \%)$ among the Chinese $(26,27)$.

$\mathrm{H}_{1}$ antihistamines down regulate allergic inflammation mainly through the $\mathrm{H}_{1}$-receptor which is widespread in smooth-muscle and neurons. $\mathrm{H}_{1}$ antihistamines are commonly classified according to function - of which, first-generation are sedating, as compared with second-generation which are relatively non-sedating. These $\mathrm{H}_{1}$ antihistamines differ from each other by their ability to cross the blood-brain barrier, which determines the presence of sedative central effects. The importance of sedation (reduction of daytime alertness, slight drowsiness or deep sleep) varies according to the degree of blood-brain barrier crossing and to the relative affinity of $\mathrm{H}_{1}$-antihistamines for peripheral and central receptors. (22) 
Harmless Over the Counter Medications- are they Truly Harmless for the Elderly?

Hydroxyzine, an $\mathrm{H}_{1}$ antagonist, is commonly prescribed for the following conditions, as adjunct treatment of anxiety, perioperative adjunct and pruritus (13). Hydroxyzine has a long $t \frac{1}{2}$ and a large volume of distribution in the elderly. Studies have also suggested the possibility of enhanced $\mathrm{H}_{1}$-receptor activity in old age (22).

Its common side effects are drowsiness (transient), xerostomia, respiratory depression (high doses) (13). It is identified in the Beers Criteria as a potentially inappropriate medication and to be avoided in patients 65 years and older (independent of diagnosis or condition) due to its potent anticholinergic properties resulting in increased risk of confusion, dry mouth, constipation, and other anticholinergic effects or toxicity; its use should also be avoided due to reduced hepatic and renal functions with advanced age and tolerance associated with use as a hypnotic (Beers Criteria [AGS 2019]). (9)

In Madam T's case, there is use of a combination of several hypnotic agents over the course of 2 months, namely benzodiazepine, hydroxyzine, zopiclone, zolpiderm (nonbenzodiazepine receptor agonists) and mirtazapine (noradrenergic and selective serotonergic antidepressant). Benzodiazepine, hydroxyzine and zopiclone are not recommended for routine use as hypnotics for the elderly due to concerns of common adverse effects such as cognitive impairment, fall risk and delirium (Beers Criteria [AGS 2019]) (9). The fact that Madam $\mathrm{T}$ still could not get a relief from her insomnia despite a combination of drugs is seemingly counterintuitive. All four drugs prescribed for Madam $\mathrm{T}$ have CNS depressant effects and their combination enhances the effects (14), which may explain the protracted delirium our patient is suffering from. However, it has been reported that all the involved drugs can cause paradoxical agitation in older patients. (28-30)

In addition, there is no advantage in combining hypnotic agents and the practice is not recommended, especially in geriatric population as there is increased fall risk. These agents, with their CNS suppressant effects, can increase the risk of fall possibly due to their delirium inducing effects, which reduce patients' safety awareness, and their negative impact on patients' psychomotor versatility. Chronic use can also result in addiction and withdrawal can lead to rebound insomnia. (23) Non-pharmacological therapy such as promoting sleep hygiene and cognitive behavioural therapy, has also been advocated as first line therapy for elderly suffering from insomnia. (24)

\section{ConClusion}

These are three cases of adverse drug reactions to analgesics, cough medication and a $\mathrm{H} 1$ antagonist in combination with other medication which have all resulted in severe unpredictable adverse outcome. The patients required prolonged hospitalization, multiple bloods tests for monitoring, sophisticated investigations and even surgery. The offending drugs implicated in Patient 1 and 3, namely NSAIDs, Anticholinergics (Cyproheptadine, Hydroxyzine) and Benzodiazepine, are well known to be associated with high risk of causing harm in the older patients. Therefore, medical care staff must pay great attention when prescribing such drugs. As for Dextromethorphan in Patient 2, the exaggerated response of the patient to the normal therapeutic dose was unpredictable. This further underlies the fact that no matter how well tolerated a drug is among the general population, using a drug always comes with a risk. Hence, pharmacotherapy should only be employed if non-pharmacological treatment is not available or inadequate. Older adults are often prescribed drugs for minor symptoms that may be better treated with nonpharmacological methods or by reviewing the current medication list to exclude usual drugs causing adverse effects. Addition of any new drugs needs to be carefully considered especially for adverse drug reactions and drug-drug interactions which may be unpredictable.

\section{REFERENCES}

[1] Qato DM et al. Use of prescription and over-thecounter medications and dietary supplements among the older adults in the United States. The Journal of American Medical Association. 2008;300:2867-2878.

[2] Jin Xiang-qiu and Min Lian-qiu. Analysis on 85 Case Reports of Adverse Drug Reactions, Afr J Tradit Complement Altern Med. 2013; 10(3): 508-512.

[3] Fu A.Z et al. Potentially inappropriate medication use and healthcvare expenditures in US community-dwelling elderly. Medical Care. 2007;45:472-476. 
Harmless Over the Counter Medications- are they Truly Harmless for the Elderly?

[4] J. Mark Ruscin and Sunny A. Linnabur, DrugRelated Problems in Older Adults, Merck Manual for Professionals

[5] Oscanoa TJ, Lizaraso F, Carvajal A. Hospital admissions due to adverse drug reactions in the elderly. A meta-analysis., Eur J Clin Pharmacol. 2017 Jun;73(6):759-770

[6] Parameswaran Nair N, Chalmers L, Bereznicki BJ, Curtain CM, Bereznicki LR. Repeat Adverse Drug Reaction-Related Hospital Admissions in Elderly Australians: A Retrospective Study at the Royal Hobart Hospital., Drugs Aging. 2017 Oct;34(10):777-783

[7] Wongrakpanich S, Wongrakpanich A, Melhado $\mathrm{K}$, Rangaswami J.A Comprehensive Review of Non-Steroidal Anti-Inflammatory Drug Use in The Elderly. Aging Dis. 2018 Feb 1;9(1):143150.

[8] Pallavi Dhanvijay, Arup K. Misra, and Sushil K. Varma., Diclofenac induced acute renal failure in a decompensated elderly patient., J Pharmacol Pharmacother. 2013 Apr-Jun; 4(2): 155-157.

[9] Beers Criteria [AGS 2019]

[10] Bryan D et al. Haemodynamically mediated Acute Renal Failure.NEJM 1986;314:97-105.

[11] A Hinsberger, V Sharma, and D Mazmanian, Cognitive deterioration from long-term abuse of dextromethorphan: a case report. J Psychiatry Neurosci. 1994 Nov; 19(5): 375-377.

[12] Jerzy L. Bem and Richard Peck, Dextromethorphan: An Overview of Safety Issue, Drug Safety, May 1992, Volume 7, Issue 3, pp 190-199

[13] Chou YC, Liao JF, Chang WY, Lin MF, Chen CF (1999) Binding of dimemorfan to sigma-1 receptor and its anticonvulsant and locomotor effects i mice, compared with Dextromethorphan and dextrophan. Brain Res 821: 516-9.

[14] Ramachander G, Williams F, Emele J (1977) Determination of dextrophan in plasma and evaluation of bioavailability ofdextromethorphan hydrobromide in humans. J Pharm Sci 66: 1047-8.

[15] Ginski M, Witkin J (1994) Sensitive and rapid behavioraldifferentiationofn-methyl-d-aspartate receptor antagonists. Psychopharmacology 114: 573-82.

[16] Bobo W, Fulton R (2004) Commentary on: severe manifestations of coricidin intoxication. Am J Emerg Med 22: 624-5.

[17] Price L, Level J (2000) Dextromethorphaninduced psychosis. Am J Psychiatry 157: 304.

[18] Banerji S, Anderson I (2001) Abuse of coriciding hbp cough and cold tables: Episodes recorded by a poison center. American Journal of HealthSystem Pharmacy 58: 1811-4.

[19] Bryner JK, Wang UK, Hui JW, Bedodo M, MacDougall C, et al. (2006) Dextromethorphan abuse in adolescence. Arch Pediatr Adolesc Med 160: $1217-22$.

[20] Simons KJ, Watson WT, Chen XY, Simons FE., Pharmacokinetic and pharmacodynamic studies of the H1-receptor antagonist hydroxyzine in the elderly. , Clin Pharmacol Ther. 1989 Jan;45(1):9-14.

[21] Touitou Y. Sleep disorders and hypnotic agents: medical, social and economical impact. Ann Pharm Fr. 2007; 65:230-238.

[22] Schroeck JL, Ford J, Conway EL, Kurtzhalts KE, Gee ME, Vollmer KA, et al. Review of Safety and Efficacy of Sleep Medicines in Older Adults. Clin Ther. 2016;38(11):2340-72

[23] Karen E. Simone., Cyproheptadine .,Critical care toxicology: Diagnosis and management of the critically poisoned patient (pp.2747-2755)

[24] Simons KJ1, Watson WT, Chen XY, Simons FE., Pharmacokinetic and pharmacodynamic studies of the H1-receptor antagonist hydroxyzine in the elderly., Clin Pharmacol Ther. 1989 Jan;45(1):9-14.

[25] Jennifer L. Schroeck Pharm D, BCPS, CGP James Ford Pharm D Erin L. Conway Pharm D Kari E.Kurtzhalts Pharm D Megan E. Gee Pharm D Krista A.Vollmer Pharm D, BCACPKari A. Mergenhagen Pharm D, Review of Safety and Efficacy of Sleep Medicines in Older Adults., Clinical Therapeutics., Volume 38, Issue 11, November 2016, Pages 2340-2372

[26] Charles M. Morin, PhD,1 Peter J. Hauri, PhD,2 Colin A.Espie, PhD,3 Arthur J. Spielman, PhD,4 
Harmless Over the Counter Medications- are they Truly Harmless for the Elderly?

Daniel J. Buysse, MD,5 and Richard R. Bootzin, PhD6, Nonpharmacologic treatment of Chronic Insomia, An American Academy of Sleep Medicine Review

[27] Dextromethorphan. Lexi-Interact. Lexicomp. Wolters Kluwer Health, Inc. Riverwoods IL

[28] Bernard, S., Neville, K. A., Nguyen, A. T., \& Flockhart, D. A. (2006). Interethnic differences in genetic polymorphisms of CYP2D6 in the US population: clinical implications. The oncologist, 11(2), 126135.

[29] Zawertailo, L. A., Kaplan, H. L., Busto, U. E., Tyndale, R. F., \& Sellers, E. M. (1998). Psychotropic effects of dextromethorphan are altered by the CYP2D6 polymorphism: a pilot study. Journal of clinical psychopharmacology, 18(4), 332-337.

[30] Schieveld, J. N., Strik, J. J., \& Bruining, H. (2018). On Benzodiazepines, Paradoxical Agitation, Hyperactive Delirium, and Chloride Homeostasis. Critical care medicine, 46(9), 1558-1559.

[31] Simons, F. E. R. (2004). Advances in H1antihistamines. New England Journal of Medicine, 351(21), 2203-2217.

[32] Rattehali R. D., Deshpande S., Jayaram M. Paradoxical agitation and sexual disinhibition following zopiclone. Prog Neurol PsychiatryCase Notes. 2009;1(1):1-4.

Citation: Vivian C. Barrera, W. T. Chang, Kuah Poh Kah, S. C. Lim. Harmless Over the Counter Medicationsare they Truly Harmless for the Elderly?. Open Journal of Geriatrics. 2019; 2(1): 18-25.

Copyright: (C) 2019 Vivian C. Barrera, W. T. Chang, Kuah Poh Kah, S. C. Lim. This is an open access article distributed under the Creative Commons Attribution License, which permits unrestricted use, distribution, and reproduction in any medium, provided the original work is properly cited. 